DOI 10.22448/AMJ.2017.3.30-30

\title{
THE RESEARCH OF QI AND BLOOD CIRCULATION METHOD FOR TREATMENT OF QI STAGNATION AND BLOOD STASIS UTERINE ADENOMYOSIS
}

\section{Feng-juan Han1 FuYang2}

1、The first hospital affiliated to Heilongjiang University of Chinese Medicine. 2、 Heilongjiang University of Chinese Medicine. E-mail:hanfengjuan2004@163.com

Abstract Based on the research of the collection of 30 patients were analyzed,Qi and blood circulation method can improve patient clinical symptoms of qi stagnation and blood stasis type adenomyosis,especially dysmenorrhea.It is high safety and non-toxic.Qi and blood circulation method can reduce the serum CA125 and anti-endometrial antibody levels.Qi and blood circulation method has no effect in improving the uterine volume.Clinical observations confirmed of uterine adenomyosis qi stagnancy and blood stasis and qi huoxue method has good clinical curative effect.

Keywords:Uterine adenomyosis;Qi stagnation and blood stasis;Qi and blood circulation;Clinical research

Uterine adenomyosis (AM) is one of the childbearing age women common hormone-dependent disorders, referring to survive in the myometrium endometrial glands and stroma, with surrounding tissue of diffuse muscle fibers or limitations of patients and the incidence of up to $21.5 \%[1]$.With ascension and younger trend in recent years,especially the prevalence of 30 to 50 years old patients and $60 \%$ of high[2].There is no uterine adenomyosis in ancient medical books of disease related records.According to its clinical manifestations can be subordinate to traditional Chinese medicine "dysmenorrhea", "menorrhagia", "remove lump", "infertility"and other areas of the disease.Think that blood stasis of TCM in mores.nay blood chamber, chongren is the main etiology and pathogenesis of uterine adenomyosis.

Objective Application and qi activating blood method in the treatment of qi and blood stasis type of uterine adenomyosis, observe changes in the patient's clinical symptoms and physical signs before and after the treatment and carry on the preliminary exploration research, evaluation and qi qi and clinical curative effect and safety of treatment of the disease, the disease to provide the reference and theoretical basis for clinical treatment.

Materials and methods

Choose from the September 2015 - December 2016 Heilongjiang Chinese Medicine college courtyard gynecology clinic patients met the criteria of 30 patients with uterine adenomyosis,give the qi and blood circulation methodof treatment of three menstrual cycles.Take itself before and after the contrast method, observe clinical curative effect, TCM symptoms before and after the treatment to improve the situation, the degree of dysmenorrhea, serum CA125, endometrial antibody levels, uterine volume changes, such as statistical analysis, evaluation of curative effect.

Results and discussion

After 3 months to make a diagnosis and give treatment, 30 cases of AM patient clinical observations indicated that the qi and blood circulation method treatment cure rate $3.33 \%$, show the efficiency of $30.00 \%$, effective rate was $40.00 \%$, total effective rate was $73.33 \%$;After treatment in patients with dysmenorrhea and other clinical symptoms such as TCM symptoms was significantly reduced.Serum CA125 and EMab were significantly reduced, the difference was statistically significant $(P<0.05)$, but the drug was uterine size has no obvious change. The clinical observation results proved that the qi and blood circulation methodis effective for AM ,and qi stagnancy and blood stasis syndrome in clinical has better clinical curative effect of total curative effect of healing.

According to modern medicine, it grinds only to AM, AM patient pathological changes mainly:Local blood rheology changes[3],The disorder of immune regulation changes and hormone level[4].This study established the qi flow, promoted blood circulation to remove blood stasis method as a treatment for the treatment of uterine adenomyosis solution qi stagnancy and blood stasis syndrome, qi operation impeded, blood stasis is AM the most important pathogenesis and foundation.

To sum up, the application of qi and blood circulation methodof uterine adenomyosis qi stagnancy and blood stasis has good clinical effect.

\section{References}

1. Campo S,Campo V,Benagiano G.Infertility and adenomyosis [J].Obstet Gynecol Int,2012:786132.

2. Lee enjoyed. Laparoscopic uterine artery block operation on joint placed Raman le the curative effect of treatment of uterine adenomyosis analysis [D]. Jinan: shandong university, 2012-9.

3. Wei Shaobin. Promoting blood circulation to remove blood stasis type traditional Chinese medicine (TCM) inhibits the multiple targets of uterine adenomyosis angiogenesis research [J]. Journal of traditional Chinese medicine clinical research, 2013, (6) : 81-82.

4. Xie Jiajun, xiao-chun Yang, promoting blood circulation to remove blood stasis drugs to study [J]. The light of traditional Chinese medicine, 2015, (4) : 887-889. 